\title{
Heavy flavour spectroscopy and exotic states at the LHC
}

\section{Roberta Cardinale ${ }^{* \dagger}$}

University and INFN Genova

E-mail: roberta.cardinaledcern.ch

The LHC, producing huge amount of $b \bar{b}$ and $c \bar{c}$ pairs, is the ideal place for spectroscopy studies which are fundamental as tests and inputs for QCD models. Many of the recently observed states, which are not fitting the standard picture, are still lacking of interpretation. A wide range of interesting spectroscopy measurements have been performed by the LHC experiments, ATLAS, $\mathrm{CMS}$ and LHCb. Only a selection of recent results are reported here. Many new and striking results are expected with the starting of the data taking in 2021 after the Long Shutdown 2 of the LHC.

XXVII International Workshop on Deep-Inelastic Scattering and Related Subjects - DIS2019 8-12 April, 2019

Torino, Italy

* Speaker.

${ }^{\dagger}$ on behalf of the LHCb Collaboration with results from ATLAS and CMS Collaborations. 


\section{Introduction}

Thanks to the large centre-of-mass energy, LHC produces a large amount of $b \bar{b}$ and $c \bar{c}$ pairs which provides great opportunities for studying production and properties of heavy flavor hadrons. ATLAS, CMS and LHCb experiments are producing many striking results in the heavy flavour spectroscopy sector. While ATLAS and CMS experiments, which are general purpose detectors, cover high $p_{T}$ region and low rapidity range, the LHCb experiment, that thanks to the excellent vertexing and particle identification capabilities is the experiment dedicated to heavy flavor, covers low $p_{T}$ range and higher rapidity region.

\section{Exotic spectroscopy}

In the last years, since 2003 with the discovery of the X(3872) state, a renovated interest in the quarkonium spectroscopy due to the observation of states with properties inconsistent with pure $c \bar{c}$ and $b \bar{b}$ states, arised. A clear pattern of the quark composition and binding mechanism of these exotic states, is still missing.

\subsection{Evidence for an $\eta_{c}(1 S) \pi^{-}$resonance in $B^{0} \rightarrow \eta_{c}(1 S) K^{+} \pi^{-}$decays}

The LHCb collaboration performed a search of exotic candidates in a fully hadronic decay channel involving the $\eta_{c}$ resonance reconstructed in the $p \bar{p}$ final state [1]. Different theoretical predictions of the existence of exotic states in the $\eta_{c} \pi^{-}$invariant mass are available $[3,6,5,4,7]$. The analysis uses Run1 and part of Run2 data for a total integrated luminosity of $4.7 \mathrm{fb}^{-1}$. Since this decay has a non-negligible non-resonant contribution, it is necessary to isolate $B^{0} \rightarrow \eta_{c} K^{+} \pi^{-}$ candidates separating them from non-resonant $B^{0} \rightarrow p \bar{p} K^{+} \pi^{-}$candidates and from combinatorial background. This is obtained performing a 2D fit, separately for Run1 and Run2, to the $m\left(p \bar{p} K^{+} \pi^{-}\right)$and $m(p \bar{p})$ invariant masses and using the SPlot technique [8]. A total yield of $\sim 1900$ candidates is obtained. An amplitude analysis is then performed. Since only pseudo-scalar particles are involved in the decay channel, a standard Dalitz plot analysis is performed using Laura++ package [9]. On the other hand, the non negligible natural width of the $\eta_{c}$ complicates the formalism. In order to parametrise the decay amplitude, an isobar model is used. In absence of exotic contributions, the $K^{* 0}$ resonances are expected to contribute to the decay amplitude. All the established $K^{* 0}$ resonance with masses within or slightly above the phase space boundary are considered. The $K^{*}$ resonances are parametrized using relativistic Breit-Wigner function except the low-mass non-resonant $K \pi$ s-wave which is parametrized using the LASS lineshape [12]. The data are not well described when including only $K^{* 0}$ resonances and in particular a discrepancy in the $\eta_{c} \pi^{-}$invariant mass is present around $4.1 \mathrm{GeV}$. A better description of the data is obtained by adding an exotic $Z_{c}^{-} \rightarrow \eta_{c} \pi^{-}$contribution as shown in Figure 1. Three quantum numbers are probed for the $Z_{c}^{-}$resonance: $J^{P}=0^{+}, 1^{-}$and $2^{+}$. The $Z_{c}^{-}$mass, width and fit fraction are measured to be $m_{Z_{c}^{-}}=4096 \pm 20_{-22}^{+18} \mathrm{MeV}, \Gamma_{Z_{c}^{-}}=152 \pm 58_{-35}^{+60} \mathrm{MeV}$ and $f_{Z_{c}^{-}}=3.3 \pm 1.1_{-1.1}^{+1.2} \%$. After considering systematic uncertainties, a $3.2 \sigma$ significance is obtained and discrimination between quantum number hypothesis is not significant. More data are needed to conclusively determine the nature of the $Z_{c}(4100)^{-}$candidate. 

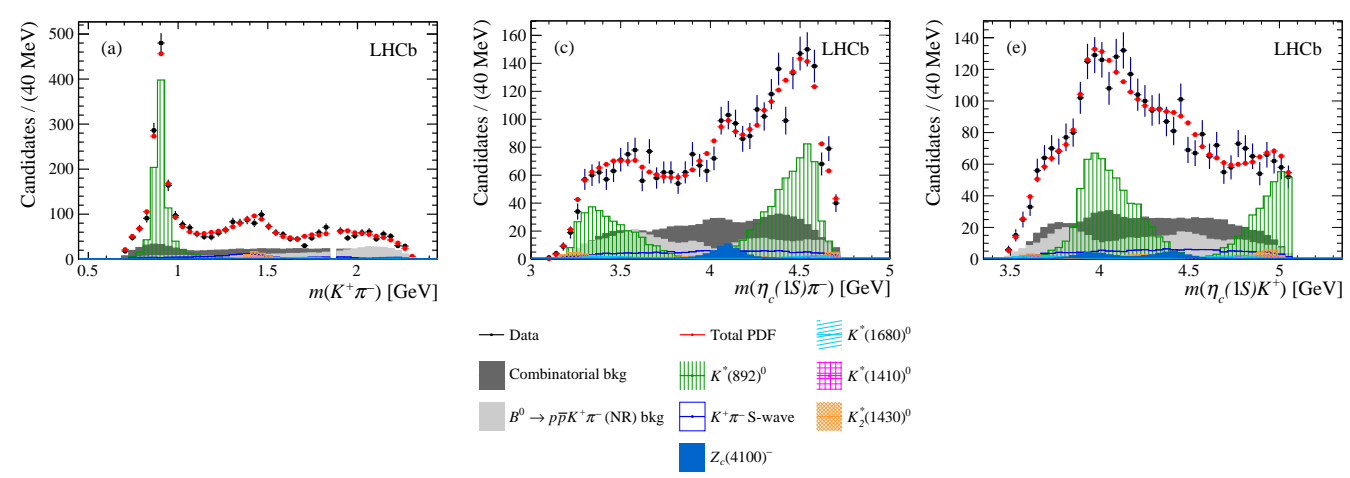

Figure 1: Projections of the data and amplitude fit including an exotic $Z_{c}^{-}$contribution onto $m\left(K^{+} \pi^{-}\right)$, $m\left(\eta_{c} \pi^{-}\right)$and $m\left(\eta_{c} K^{+}\right)$

\subsection{Exotic contributions to $B^{0} \rightarrow J / \psi K^{+} \pi^{-}$}

Belle Collaboration observed an exotic state $Z_{c}(4200)^{-}$decaying to $J / \psi \pi^{-}$[10]. BaBar, from a model independent analysis, concluded that the $J / \psi \pi^{-}$mass spectrum is well described using only known $K^{* 0}$ resonances without including any exotic contribution [11]. LHCb performed an angular analysis using the full Run1 data corresponding to an integrated luminosity of $3 \mathrm{fb}^{-1}$ [13]. The total available yield is a factor 20 and 40 larger than Belle and BaBar dataset, respectively. A model independent 4D analysis is performed based on an expansion in angular moments only having minimal assumptions on the $K^{* 0}$ spectrum to avoid large systematic uncertainties due to the poor knowledge of the $K^{* 0}$ spectrum. The comparison between background-subtracted data and weighted simulated events show that $J_{\max }^{k}=2$ is not describing the two peaking structures at 4200 $\mathrm{MeV}$ and $4600 \mathrm{MeV}$ (see Figure 2). The $K^{*}$-only hypothesis is rejected with a significance of $10 \sigma$.
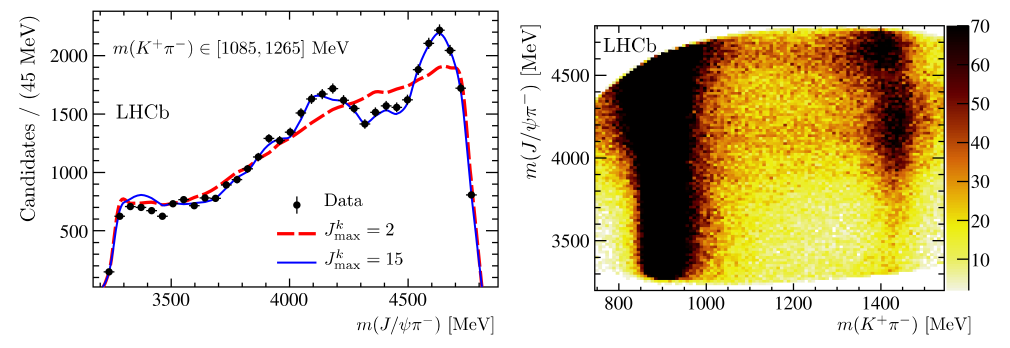

Figure 2: Comparison of $m\left(J / \psi \pi^{-}\right)$between the background-subtracted data and simulated events weighted by moments models with $J_{\max }^{k}=2$ and $J_{\max }^{k}=15$ (left) and background -subtracted 2D distribution of $m\left(J / \psi \pi^{-}\right)$vs $m\left(K^{+} \pi^{-}\right)$where two structures are visible around $m\left(J / \psi \pi^{-}\right) \sim 4200 \mathrm{MeV}$ and $\sim 4600 \mathrm{MeV}$.

An amplitude analysis is necessary in order to correctly interpret these structures.

\subsection{Pentaquarks}

One of the striking news in the spectroscopy sector was the observation of significant pentaquark structures in $\Lambda_{b}^{0} \rightarrow J / \psi p K^{-}$decays. A model independent analysis and a $6 \mathrm{D}$ amplitude fit were performed $[14,15]$ and two exotic states, $P_{c}(4450)^{+}$and $P_{c}(4380)^{+}$, with opposite parities 
were found. An update is performed at $\mathrm{LHCb}$ using both Run1 and Run2 data corresponding to an integrated luminosity of $9 \mathrm{fb}^{-1}$ [16] with a signal yield an order of magnitude larger. A narrow peak around $4.3 \mathrm{GeV}$ is observed and the previously reported structure at $4.45 \mathrm{GeV}$ appears as two narrow peaks. The three peaks are much more visible when requiring $m_{K p}>1.9 \mathrm{GeV}$ removing the dominant $\Lambda^{*}$ backgrounds as shown in Figure 3. Since the observed peaks are narrow, a
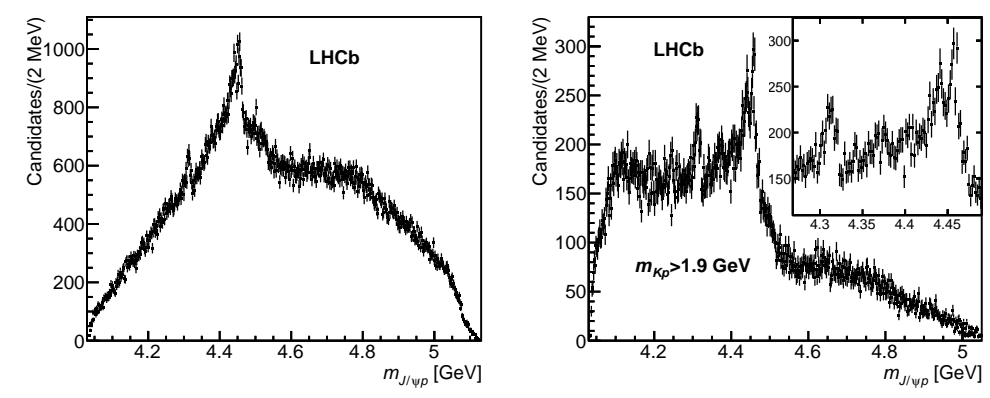

Figure 3: Distribution of $m_{J / \psi p}$ from $\Lambda_{b}^{0} \rightarrow J / \psi p K^{-}$candidates (left) and after suppression of the dominant $\Lambda^{*} \rightarrow p K^{-}$contribution requiring $m_{K p}>1.9 \mathrm{GeV}$ (right). The inset shows a zoom into the region of the narrow $P_{c}^{+}$peaks.

1D fit is performed obtaining $M\left(P_{c}(4312)^{+}\right)=\left(4311.9 \pm 0.7_{-0.6}^{+6.8}\right) \mathrm{MeV}, \Gamma\left(P_{c}(4312)^{+}\right)=(9.8 \pm$ $\left.2.7_{-4.5}^{+3.7}\right) \mathrm{MeV}, M\left(P_{c}(4440)^{+}\right)=\left(4440.3 \pm 1.3_{-4.7}^{+4.1}\right) \mathrm{MeV}, \Gamma\left(P_{c}(4440)^{+}\right)=\left(20.6 \pm 4.9_{-10.1}^{+8.7}\right) \mathrm{MeV}$ and $M\left(P_{c}(4457)^{+}\right)=\left(4457.3 \pm 0.6_{-1.7}^{+4.1}\right) \mathrm{MeV}, \Gamma\left(P_{c}(4457)^{+}\right)=\left(6.4 \pm 2.0_{-1.9}^{+5.7}\right) \mathrm{MeV}$. Proximity of the $\Sigma_{c}^{+} \bar{D}^{0}$ and $\Sigma_{c}^{+} \bar{D}^{* 0}$ thresholds to the observed narrow peaks suggests that they play an important role in the dynamics of these states.

\section{Standard spectroscopy}

\subsection{Near-threshold $D \bar{D}$ spectroscopy and observation of a new charmonium state}

The near-threshold $D \bar{D}$ mass spectra analysis, performed at LHCb, uses full Run1 and Run2 data corresponding to an integrated luminosity of $9 \mathrm{fb}^{-1}$ [17]. Promptly produced $D^{+} D^{-}$and $D^{0} \bar{D}^{0}$ pairs are selected. To better describe the background, the fit, shown in Figure 4, is performed in three different overlapping mass regions. A new narrow charmonium state, $X(3842)$,
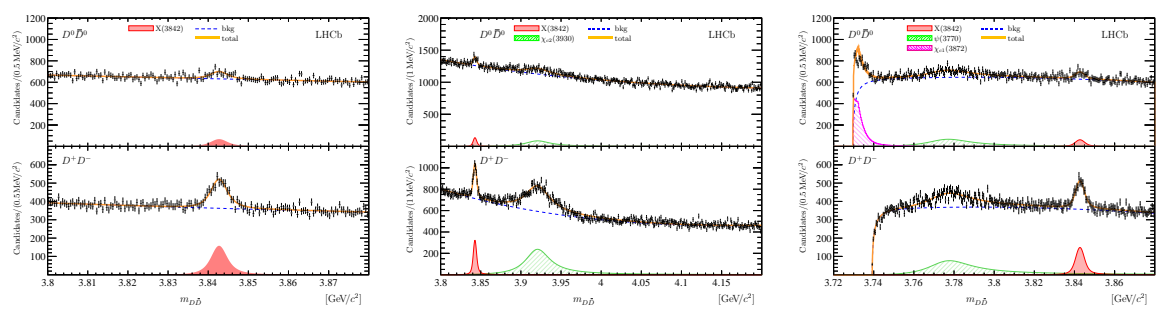

Figure 4: Mass spectra of (top) $D^{0} \bar{D}^{0}$ and (bottom) $D^{+} D^{-}$candidates in the narrow $(3.80-3.88 \mathrm{GeV})$ region (left), high-mass region (3.8-4.2 GeV) (middle) and low-mass near-threhold region $(<3.88 \mathrm{GeV})$.

is visible and its mass and width are measured to be $m=3842.71 \pm 0.16 \pm 0.12 \mathrm{MeV}$ and $\Gamma=$ 
$2.79 \pm 0.51 \pm 0.35 \mathrm{MeV}$. It can be interpreted as the $\psi_{3}\left(1^{3} D_{3}\right)$ state with $J^{P C}=3^{--}$. In addition, the hadroproduction of $\psi(3770)$ and of $\chi_{c 2}(3930)$ are observed for the first time. For the $\chi_{c 2}(3930)$, the measured mass, $m\left(\chi_{c 2}(3930)\right)=3921.9 \pm 0.6 \pm 0.2 \mathrm{MeV}$, is $2 \sigma$ lower then the world average being midway the expected mass for this state and for the $X(3915)$ state. Further studies are necessary to understand if they are two distinct states.

\section{$3.2 B_{c}$ mesons}

$B_{c}$ mesons are quite unique in the Standard Model since they are formed by two different heavy quarks. Beyond the ground state, a rich spectrum is expected. On the other hand, experimental observations are just a few since the production cross-section is small and there are large backgrounds. All the states below the $B D$ threshold, such as the $B_{c}(2 S)^{+}$and the $B_{c}^{*}(2 S)^{+}$can only decay via radiative or pionic transitions. In particular the main decay modes are $B_{c}(2 S) \rightarrow$ $B_{c} \pi^{+} \pi^{-}$and $B_{c}^{*}(2 S) \rightarrow B_{c}^{*}\left(\rightarrow B_{c} \gamma\right) \pi^{+} \pi^{-}$where the low energy photon is not reconstructed. Both states can be then observed reconstructing $B_{c} \pi^{+} \pi^{-}$with the $B_{c}^{*+}(2 S)$ at a mass $M\left(B_{c}^{+}(2 S)\right)_{\text {rec }}=$ $M\left(B_{c}^{+}(2 S)\right)-M\left(B_{c}^{*+}\right)-M\left(B_{c}^{+}\right)$, lower than the $B_{c}(2 S)^{+}$state. The first observation of an excited $B_{c}^{+}$state has been performed at ATLAS using the full Run1 data [18]. A peak with a 5.2 $\sigma$ has been observed and the measured mass $M=(6842 \pm 4 \pm 5) \mathrm{MeV}$ is consistent with expectations of the $B_{c}(2 S)$ mass. But since the mass predictions for the $B_{c}(2 S)$ and the $B_{c}(2 S)^{*}$ differ by about 20$50 \mathrm{MeV}$ and since the soft photon escapes identification, the observed peak can be a superposition of the two states. The analysis of the excited $B_{c}^{+}$states performed at the CMS experiment uses the full Run2 data sample corresponding to an integrated luminosity of $140 \mathrm{fb}^{-1}$ [19]. The two peaks which are identified as the $B_{c}(2 S)$ and the $B_{c}(2 S)^{*}$, are well separated and have significance greater than $5 \sigma$ with $67 \pm 10$ and $51 \pm 10$ events for the lower-mass and the higher-mass peak, respectively. The two states observed by CMS have been confirmed recently by LHCb [20]. The analysis uses $8.5 \mathrm{fb}^{-1}$ of Run1 and Run2 data. The significances are of greater than $5 \sigma$ for the $B_{c}(2 S)^{*}$ and $2.2 \sigma$ for the $B_{c}(2 S)$. Mass measurements are the most precise up to date and are compatible with CMS results. The excited $B_{c}$ mass spectra are shown in Figure 5.
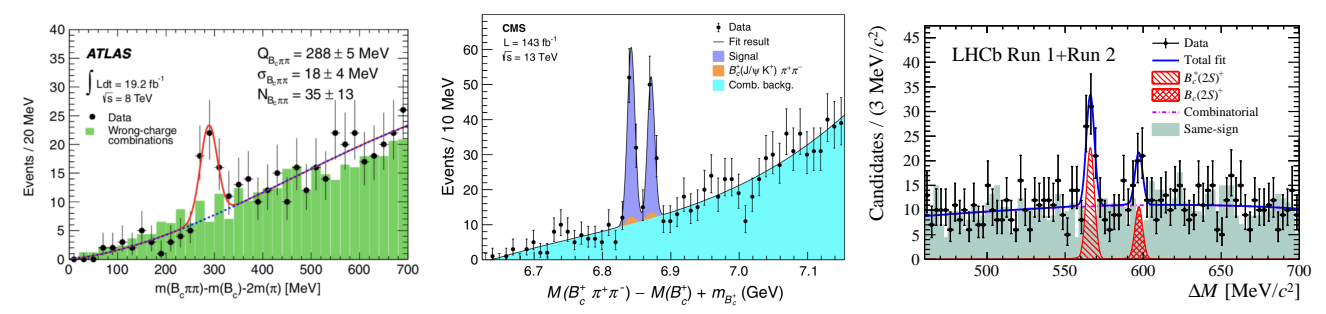

Figure 5: The excited $B_{c}$ mass spectra from ATLAS (left), CMS (centre) and LHCb (right).

\section{Conclusions}

Thanks to the large cross-section and to the excellent performance of the detectors, the LHC experiments are producing many interesting results in the spectroscopy sector. Long Shutdown 2 started and the detectors are going to be upgraded to be able to collect a larger data sample with high efficiency and better performance starting in 2021. 


\section{References}

[1] R. Aaij et al. [LHCb Collaboration], Eur. Phys. J. C 78 (2018) no.12, 1019 doi:10.1140/epjc/s10052-018-6447-z [arXiv:1809.07416 [hep-ex]].

[2] M. Ablikim et al. [BESIII Collaboration], Phys. Rev. Lett. 110 (2013) 252001 doi:10.1103/PhysRevLett.110.252001 [arXiv:1303.5949 [hep-ex]].

[3] M. B. Voloshin, Phys. Rev. D 87 (2013) no.9, 091501 doi:10.1103/PhysRevD.87.091501 [arXiv:1304.0380 [hep-ph]].

[4] E. Braaten, Phys. Rev. Lett. 111 (2013) 162003 doi:10.1103/PhysRevLett.111.162003 [arXiv:1305.6905 [hep-ph]].

[5] L. Liu et al. [Hadron Spectrum Collaboration], JHEP 1207 (2012) 126 doi:10.1007/JHEP07(2012)126 [arXiv:1204.5425 [hep-ph]].

[6] G. K. C. Cheung et al. [Hadron Spectrum Collaboration], JHEP 1612 (2016) 089 doi:10.1007/JHEP12(2016)089 [arXiv:1610.01073 [hep-lat]].

[7] L. Maiani, F. Piccinini, A. D. Polosa and V. Riquer, Phys. Rev. D 71 (2005) 014028 doi:10.1103/PhysRevD.71.014028 [hep-ph/0412098].

[8] M. Pivk and F. R. Le Diberder, Nucl. Instrum. Meth. A 555 (2005) 356 doi:10.1016/j.nima.2005.08.106 [physics/0402083 [physics.data-an]].

[9] J. Back et al., Comput. Phys. Commun. 231 (2018) 198 doi:10.1016/j.cpc.2018.04.017 [arXiv:1711.09854 [hep-ex]].

[10] K. Chilikin et al. [Belle Collaboration], Phys. Rev. D 90 (2014) no.11, 112009 doi:10.1103/PhysRevD.90.112009 [arXiv:1408.6457 [hep-ex]].

[11] B. Aubert et al. [BaBar Collaboration], Phys. Rev. D 79 (2009) 112001 doi:10.1103/PhysRevD.79.112001 [arXiv:0811.0564 [hep-ex]].

[12] D. Aston et al., Nucl. Phys. B 296 (1988) 493. doi:10.1016/0550-3213(88)90028-4

[13] R. Aaij et al. [LHCb Collaboration], Phys. Rev. Lett. 122 (2019) no.15, 152002 doi:10.1103/PhysRevLett.122.152002 [arXiv:1901.05745 [hep-ex]].

[14] R. Aaij et al. [LHCb Collaboration], Phys. Rev. Lett. 115 (2015) 072001 doi:10.1103/PhysRevLett.115.072001 [arXiv:1507.03414 [hep-ex]].

[15] R. Aaij et al. [LHCb Collaboration], Phys. Rev. Lett. 117 (2016) no.8, 082002 doi:10.1103/PhysRevLett.117.082002 [arXiv:1604.05708 [hep-ex]].

[16] R. Aaij et al. [LHCb Collaboration], Phys. Rev. Lett. 122 (2019) no.22, 222001 doi:10.1103/PhysRevLett.122.222001 [arXiv:1904.03947 [hep-ex]].

[17] R. Aaij et al. [LHCb Collaboration], arXiv:1903.12240 [hep-ex].

[18] G. Aad et al. [ATLAS Collaboration], Phys. Rev. Lett. 113 (2014) no.21, 212004 doi:10.1103/PhysRevLett.113.212004 [arXiv:1407.1032 [hep-ex]].

[19] A. M. Sirunyan et al. [CMS Collaboration], Phys. Rev. Lett. 122 (2019) no.13, 132001 doi:10.1103/PhysRevLett.122.132001 [arXiv:1902.00571 [hep-ex]].

[20] R. Aaij et al. [LHCb Collaboration], Phys. Rev. Lett. 122 (2019) no.23, 232001 doi:10.1103/PhysRevLett.122.232001 [arXiv:1904.00081 [hep-ex]]. 